Conclusions The authors recommend for this population to use a cut-off level of $20 \mathrm{mg} / \mathrm{L}$ to start antibiotics.

\section{USEFULNESS OF DELTA NEUTROPHIL INDEX FOR ASSESSING NEONATAL SEPSIS}

doi:10.1136/archdischild-2012-302724.1194

SM Lee, HS Eun, R Namgung, MS Park, KI Park, C Lee. Pediatrics, Yonsei University College of Medicine, Seoul, Republic of Korea

Objectives Early detection and aggressive treatment for neonatal sepsis is important for survival. This study investigated the significance of calculated delata neutrophil index (DNI) as a prognostic factor of severe sepsis.

Methods In a retrospective study, 72 neonates admitted to Severance Children's Hospital and Gangnam Severance Hospital between Jan 2009. to Dec 2010, were recruited. Twenty four infants were diagnosed as blood proven sepsis, and 48 neonates matched for gestation were recruited as controls. Among 24 infants, 5 patients died within 7 days.

Results In univariate analysis, mean DNI (at diagnosis, after $24 \mathrm{hr}$ and $72 \mathrm{hr}$ ), C-reactive protein and WBC for sepsis group were significantly higher, and neutrophil count, platelet count were significantly lower than control groups. Among sepsis group, mean DNI at diagnosis ( 6.5 vs $3.7 \mathrm{p}=0.048)$, DNI at $72 \mathrm{hr}$ ( 8.4 vs $2.1, \mathrm{p}=0.003$ ) CRP at $72 \mathrm{hr}$ ( 67 vs $21, \mathrm{p}=0.010)$ and platelet count ( 85000 vs 141000 , $\mathrm{p}=0.008$ ) for patient with mortality were significantly increased compared to the patients with survival. Other demographic factors are not remarkable. In multiple logistic regression analysis, mortality in sepsis significantly correlated with DNI at $72 \mathrm{hr}$, odds ratio (OR) 1.47, 95\% confidence interval (CI) 1.1-5.6 ( $\mathrm{p}=0.032$ ), and with platelet, OR $0.93,95 \%$ CI $0.51-0.99$ ( $p=0.014$ ). In ROC analysis, provided DNI at $72 \mathrm{hr}$ at cut off value of $12 \%$ predicted mortality with $81 \%$ sensitivity and $87 \%$ specificity.

Conclusion DNI can have implications for sepsis and may be valuable to assess the prognosis of patient with sepsis.

\section{INFLUENCE OF PROCALCITONIN (PCT) LEVELS ON THE DIRECTION OF THE THERAPY OF PERINATAL INFECTIONS}

doi:10.1136/archdischild-2012-302724.1195

G Tálosi, A Gajda, K Mader, J Kiss, S Túri. Department of Pediatrics, University of Szeged, Szeged, Hungary

Background and Aims One the main causes of perinatal mortality is infection. PCT measurement is regularly performed in our clinical practice. Along with the clinical condition, PCT level is an important factor in the decision of therapeutical interventions. Depending on the condition, immunoglobulin and pentoxifylline administration is considered. We wanted to examine, how much our therapeutical decisions were influenced by the PCT levels measured.

Methods We analyzed retrospectively the data of the neonates admitted in 2011 to our tertiary Neonatal Intensive Care Unit. PCT was measured routinely at the age of 16-32 hours.

Results Depending on the severity of the clinical condition and the PCT levels empiric antibiotic treatment (1st group; $34.5 \pm 4.6$ gestational weeks, $2435 \pm 892 \mathrm{~g} ; \mathrm{n}=29$ ), antibiotic therapy plus pentoxifylline (2nd group; 35.7 \pm 3.8 gestational weeks, 2515 $\pm 858 \mathrm{~g} ; \mathrm{n}=27$ ) and antibiotics plus intravenous immunoglobulin with or without pentoxifylline (3rd group; 33.6 \pm 3.5 weeks; $2211 \pm 851$ g; $n=26$ ) were given. There was no significant difference between the groups, regarding the gestational ages and birthweights. There was a significant difference among the three groups, regarding the PCT levels at the age of $18-32$ hours: $(8.7 \pm 4.2 ; 23.3 \pm 18.4$ and $34.3 \pm 219 \mathrm{ng} / \mathrm{ml})$. There was a notable decrease of the PCT levels in every group.
Conclusions Although the dynamics of PCT show a pronounced difference, compared to the later life, with an adequate evaluation along with the clinical status it is an important tool in the diagnostics of perinatal infections. Its analysis together with the application of the immunomodulant pentoxifylline therapy may decrease the use of immunoglobulins.

\section{NORMAL VALUES OF C REACTIVE PROTEIN IN TERM BABIES}

doi:10.1136/archdischild-2012-302724.1196

${ }^{1} \mathrm{C}$ Bellieni, ${ }^{2} \mathrm{G}$ Buonocore. ${ }^{1}$ Pediatrics and Obstetrics, ${ }^{2}$ University of Siena, Siena, Italy

Aim To assess the normal values of $C$ reactive Protein (CRP) in our population. We included only those babies in which we had no retrospective doubt of having no infection.

Material and Methods We studied all babies born in the year 2010 in the Siena hospital, in whom PCR was measured for the risk of neonatal infection. we excluded from this retrospective analysis the following babies: Gestational age $<38$ weeks, positive hemocolture, high blood cell count, antibiotic treatment already begun before CRP determination.

Results Results are reported as follows: a-Babies (n) b-Mean CRP value (mg\%) c-SD. Before 12 hours form birth: babies n99, CRP0.14, SD0.20. At 24 hours babies n92, CRP0.42, 0.61. At 36 hours n8, CRP0.38, SD0.56. At 48 hours babies n349, CRP0.44, SD0.51. At 72 hours babies n145, CRP0.31, SD0.38. At 96 hours babies n52, CRP0.20, SD0.30. At 108 hours n9, CRP0.05, SD0.10At 120 hours babies n4, CRP0.06, SD0.06.

Conclusion These date are useful to have CRP normality parameters in the newborn: they confirm that in the first hours of life, CRP values are far higher than those of the adults.

\section{ROLE OF LABORATORY TEST IN NEONATAL SEPSIS DIAGNOSIS}

doi:10.1136/archdischild-2012-302724.1197

N Kolici. Obstetric-Gynecologic University Hospital Nr 2 Tirane, Tirana, Albania

Background Early detection of sepsis in neonate is one of the most difficult problems facing neonatal care providers and clinicians today. The ability to early diagnosis or rule out neonatal sepsis results in to limit inappropriate antibiotic exposure and lowering the cost of therapy.

This study was conducted to determine the value of some laboratory test in early detection for neonatal septicemia. Besides this, we wish to know the comment causal organisms for neonatal sepsis in our situations.

Aim To determine if any laboratory tests can predict neonatal sepsis prior to positive blood culture.

Method Is a cohort prospective study. Rule in, admitted children ages $<28$ d in our NICU during 2011, with suspected infection. Based on clinical and biological findings, diagnoses were categorized in: A: proven sepsis(positive blood culture) B: probable sepsis(negative blood culture but laboratory consist with sepsis. C: clinically sepsis without any positive culture or laboratory abnormalities.

The validity of laboratory tests which had performed as sepsis work-up, were compared against positive blood culture as gold standard test.

Results The most common causative organisms were E.coli (50\%). Among laboratory tests, CRP had the best sensitivity(84.12\%) and negative predictive value $(91.3 \%)$, but poor positive predictive value $(59.5 \%)$, the specificity of it was $74.46 \%$. WBC, $\mathrm{I} / \mathrm{T}>0.2$, and segments $>10 \%$ have high specificity to rule out sepsis.

Conclusion No laboratory tests alone can be used as early detection of septicemia accurately. 


\section{PERIPHERALLY INSERTED CENTRAL VENOUS CATHETERS IN NEONATE: A QUICK AND EFFICIENT STANDARDIZED TECHNIQUE}

doi:10.1136/archdischild-2012-302724.1198

S Breinig, S Pelluau, K Laganthe, MC Granier, M Gineste, C Alberge, MC Bloom, MO Marcoux. Intensive Care Unit, Hopital des Enfants, Toulouse, France

Background and Aims Peripherally inserted central venous catheters (PICCs) are commonly used for neonatal vascular access. Early catheter-related sepsis and catheter non infectious complications are linked with initial dressing method. We evaluate efficacy of our specific PICCs insertion protocol with trained nurses and doctors.

Methods We observed prospectively 1686 PICC procedures in neonate (0-28d) from 2002 to 2011. Silicon PICCs were inserted from 0 to 28 days corrected age. Procedure followed our specific protocol. Results 1686 PICCs were attempted with a success rate of $96.2 \%$ in neonates with a mean gestational age of 29.7 weeks at a median age of $3(0-94 \mathrm{~d})$ days of life.

In the successful PICCs, median number of venous puncture was 1 (1-13), median time spent was 20 (5-120) minutes, device change in $11.7 \%$ and site change in $5.9 \%$. Median temperature difference between the beginning and the end of the procedure was very low: $-0.2^{\circ} \mathrm{C}$ ( -1.5 to 1.8$) .187$ complications (11.5\%) occurred: 105 diffusions of which 6 pericardic effusions, 25 occlusions, 37 porous catheters, 7 surrounding catheter tissue inflammations. 13 PICCs were removed for infection (sepsis or local mycosis).

Conclusions Standardized protocol with specific nurse and doctor leads to a success rate of $96.2 \%$ with a small time spent for insertion and a median of one attempt mostly in the initial chosen site. This quick method leads to low neonate cooling and expose patients to minimal infection risk and complications.

\section{METABOLIC RISK FACTORS AND PROGNOSIS IN CHILDREN WITH UROLITHIASIS}

doi:10.1136/archdischild-2012-302724.1199

${ }^{1} \mathrm{~S}$ Yüksel, ${ }^{2} \mathrm{H}$ Tancer Elçi, ${ }^{3} \mathrm{~T}$ Ince Becerir, ${ }^{2} \mathrm{M}$ Deniz, ${ }^{4} \mathrm{~A}$ Koçyiğit, ${ }^{5} \mathrm{~A}$ Ergin. ${ }^{1}$ Pediadric Nephrology; ${ }^{2}$ Pediatrics; ${ }^{3}$ Pediatric Nephrology; ${ }^{4}$ Radiodiagnostics; ${ }^{5}$ Public Health Pamukkale University, Faculty of Medicine, Denizli, Turkey

Objective To investigate the demographic characteristics, clinical features, and metabolic risk factors of children with urolithiasis.

Methods This retrospective study enrolled 52 boys, 51 girls with urolithiasis diagnosed by ultrasonography. Mean age at presentation was 60(1-192) months, and mean follow-up period was 5.5 (1-27)months.

Results The most common symptom was restlessness in infants (<1year), while it was abdominal or flank pain in older children $(p<0.001)$. Microcalculi (stone diameter $<3 \mathrm{~mm})$ and calculi $(>3 \mathrm{~mm})$ were found in $26 \%$ and in $74 \%$ of patients, respectively. Hypercalciuria was the most common abnormality, followed by hypomagnesiuria (Table 1). Recurrent urinary tract infection (UTI) was detected in half of the patients. Four patients underwent ESWL, five underwent open surgery, and the other 94 were treated with conservative therapies. Spontaneous passage occurred in 19 patients. Stone analysis revealed calcium-oxalate in $85 \%$. At the time of their last visit, in $70 \%$ of the patients with conservative therapies, the stones were disappeared or diminished in size by appropriate therapy such as water intake, diet, hydrochlorothiazide and potassium-citrate.

Conclusion Identifying the underlying metabolic risk factor is important in order to choose the appropriate treatment modality, prevent stone recurrence and renal damage. Patients presenting with restlessness, especially infants must be evaluated in terms of renal stone disease by ultrasonography.

\section{THE CORRELATION BETWEEN RENAL DAMAGE AND CLINICAL AND LABORATORY FINDINGS IN CHILDREN WITH ACUTE PYELONEPHRITIS}

doi:10.1136/archdischild-2012-302724.1200

${ }^{1} \mathrm{~S}$ Yüksel, ${ }^{2} \mathrm{~B}$ Seyhan, ${ }^{1} \mathrm{~T}$ Becerir, ${ }^{3} \mathrm{~A}$ Koçyiğit, ${ }^{4} \mathrm{D}$ Yüksel. 'Department of Pediatric Nephrology, Pamukkale University School of Medicine; 'Tavas State Hospital ${ }^{3}$ Department of Radiodiagnostic; ${ }^{4}$ Department of Nuclear Medicine, Pamukkale University School of Medicine, Denizli, Turkey

Objective The aim of this study was to determine the risk factors such as age, sex, duration of fever, voiding dysfunction, constipation, history of recurrent UTI, the levels of C-reactive protein (CRP), erythrocyte sedimentation rate (ESR), white blood cell (WBC), polymorphonuclear leukocytes (PNL), platelet count, mean platelet volume $(M P V)$, serum urea, creatinine, proteinuria, type of microorganisms, presence of vesicoureteral reflux(VUR) for the development of renal damage in patients with acute pyelonephritis

Material and Methods A total of 197 children (44 boys, 153 girls) were enrolled in the study, mean age was $4.7 \pm 4.0$ years ( 1 month-16 years). Ultrasonography, renal cortical scintigraphy (RCS) with ${ }_{99 \mathrm{~m}} \mathrm{Tc}$-Dimercaptosuccinic acid and voiding cystourethrography were performed in all patients. The patients were grouped by age according to presumed risk of renal damage: high risk( $\leq 1$ year), moderate risk( $1-5$ years), and low risk ( $>5$ years).

Results Renal lesions on RCS were detected in 91 patients. Abnormal RCS were found in 35\% of infants younger than 1 year, in $57 \%$ of children between 1-5 years and in $42 \%$ of children older than 5 years. Abnormal RCS was found in $66 \%$ of the children with VUR and in $42.7 \%$ of those without VUR $(p=0.005)$. There was a significant positive correlation between abnormal RCS and VUR, duration of fever ( $>2$ days), history of recurrent UTI, high levels of ESR, CRP, WBC, and PNL at the presentation.

Conclusion The patients with VUR were 2.6 times more likely to have renal damage. The risk of renal damage should be considered in all age groups.

\section{WHOLE EXON DELETION IN THE CLAUDIN 16 GENE, A NOVEL MUTATION IN FAMILIAL HYPOMAGNESEMIA/ HYPERCALCIURIA/NEPHROCALCINOSIS (FHHNC) AND SENSORINEURAL DEAFNESS (SND)}

doi:10.1136/archdischild-2012-302724.1201

${ }^{1}$ SA Sanjad, ${ }^{2} \mathrm{Y}$ Lu, ${ }^{1,3} \mathrm{C}$ Khoury, ${ }^{4} \mathrm{Z}$ Habbal, ${ }^{2} \mathrm{R}$ Lifton, Family with FHHNCS AND. ${ }^{1}$ Pediatrics, American University of Beirut Medical Center, Beirut, Lebanon; ${ }^{2}$ Genetics, Yale University School of Medicine, New Haven, CT; 3/nternal Medicine, Northwestern University, Chicago, IL, USA; ${ }^{4}$ Pathology, American University of Beirut Medical Center, Beirut, Lebanon

Abstract 1199 Table 1 Urine metabolic analysis

\begin{tabular}{|c|c|c|c|c|c|c|c|}
\hline Risk factor & Patients(\%) & Risk factor & Patients(\%) & Risk factor & Patients(\%) & Risk factor & Patients(\%) \\
\hline Hypercalciuria & $32(31.1)$ & Cystinuria & $4(3.9)$ & Hyperoxaluria & $2(1.9)$ & Hypercalciuria+hypernatriuria & $2(1.9)$ \\
\hline Hypomagnesuria & $13(12.6)$ & Hyperuricosuria & $3(2.9)$ & Hypercalciuria+hypocitraturia & $4(3.9)$ & Hypocitraturia+hypomagnesuria & $2(1.9)$ \\
\hline Hypernatriuria & $11(10.7)$ & Hypocitraturia & $3(2.9)$ & Hypercalciuria+hyperoxaluria & $3(2.9)$ & Normal & $24(23.3)$ \\
\hline
\end{tabular}

